\title{
An enigmatic trio of Klinefelter's syndrome, autoimmune hypothyroidism and nephrotic syndrome
}

\author{
A Rao' ${ }^{1}$, J Jain ${ }^{2}, \mathrm{HK}_{\text {Aggarwal }}^{3}$, P Jain ${ }^{4}$
}

Klinefelter's syndrome is the most common chromosomal disorder associated with testicular dysfunction and male infertility. Those affected by Klinefelter's syndrome are at increased risk of systemic lupus erythematosus, breast cancer, non-Hodgkin's lymphoma, and lung cancer. Nephrotic syndrome in association with Klinefelter's syndrome has never been reported in the literature.

Keywords: autoimmune hypothyroidism, Klinefelter's syndrome, proteinuria, nephrotic syndrome

Declaration of interests: No conflict of interests declared

\author{
Correspondence to: \\ D Jain \\ Department of Medicine \\ Pt. B.D. Sharma University \\ of Health Sciences, \\ ROHTAK-124001 (Haryana) \\ India
}

Email:

jaindeepakdr@gmail.com

\section{Case report}

A 28-year-old male presented to the outpatient department with a history of facial puffiness and lower limbs swelling of two weeks' duration. The patient noted puffiness of face initially, mainly over the peri-orbital areas, more obvious during the morning and decreasing as the day progressed. He also noted bilateral pedal oedema, which was initially present on the ankles, gradually progressing up to his knees. There was no history of urinary or bowel disturbances, appetite or weight loss, abdominal pain or distension, chest pain, palpitations, previous blood transfusion, drug intake or high risk behaviour. There was no history of tuberculosis, cardiac illness, diabetes or hypertension.

The patient had been married for six years and had no live children. He had previously been investigated and found to have a sexual developmental disorder and he had late onset puberty.

At admission, he had a body mass index of $28.65 \mathrm{~kg} / \mathrm{m}^{2}$. His blood pressure was 148/90 (19.68/11.97 kPa). General physical examination revealed thick, coarse scalp hair, a pitting type of pedal oedema and facial puffiness. He had a eunuchoid appearance with length of lower segment (1.02 $\mathrm{m})$ greater than upper segment $(0.82 \mathrm{~m})$, arm span (1.97 $\mathrm{m})$ greater than height $(1.84 \mathrm{~cm})$, waist to hip ratio $1: 1$, palpable glandular breast tissue of $0.06 \mathrm{~m}$ around the nipple, small, firm testes with a volume of $10^{-6} \mathrm{~m}^{3}$ and stretched penile length of $0.12 \mathrm{~m}$ (Figure 1). Tanner's score indicated the patient's pubic and genital developmental age to be around thirteen years. His intelligence quotient was 93. The rest of the systemic examination, including cardiovascular, respiratory, abdomen and nervous system, were within normal limits.

The laboratory examination revealed normal haematological and liver parameters. Serum creatinine was $1.7 \mathrm{mg} / \mathrm{dL}(0.7-$ 1.3). Urine examination revealed $4+$ albumin with negative casts; 24 hour urine collection showed a proteinuria of 3.14 $\mathrm{g} /$ day. His random blood sugar was $93 \mathrm{mg} / \mathrm{dL}$. Viral serology for hepatitis and HIV was negative as were tests for ANA and dsDNA.

His hormonal profile showed increased serum FSH (88.93 $\mathrm{mIU} / \mathrm{mL}), \mathrm{LH}(25.5 \mathrm{mlU} / \mathrm{mL})$, and oestradiol $(50.30 \mathrm{pg} / \mathrm{mL})$ levels, and decreased serum testosterone level (42.91 $\mathrm{ng} / \mathrm{dL}$ ) suggestive of hypergonadotropic hypogonadism. Thyroid profile showed decreased fT3 $(0.97 \mathrm{pg} / \mathrm{mL})$ and fT4 (0.65 ng/dL), increased TSH $(100 \mathrm{mlU} / \mathrm{mL})$ and positive anti TPO antibodies (152.1 IU/mL) indicating autoimmune hypothyroidism.

His semen analysis revealed azoospermia with a sperm count of $<10,000 / \mathrm{mL}$, sperm motility of $<10 \%$ and $<1 \%$ normal forms. Cytogenetic analysis of PHA stimulated peripheral lymphocytes revealed an extra $X$ chromosome with karyotype of 47 XXY (Figure 2).

Ultrasound showed enlargement of the thyroid gland with altered echotexture suggestive of thyroiditis. The DEXA scan of the lumbar spine revealed osteopenia with a $T$ score of -2.3 . The remainder of the radiological investigations including ultrasonography of the abdomen and chest X-ray was normal.

\footnotetext{
${ }^{1}$ Senior Resident, Department of Medicine, Kempegowda Institute of Medical Sciences, Bangalore, India; ${ }^{2}$ Associate Professor, Department of Medicine; ${ }^{3}$ Senior Professor \& Head, Department of Medicine IV; ${ }^{4}$ Assistant Professor, Department of Pathology, Pt. B. D. Sharma University of Health Sciences, Rohtak, India
} 
Figure 1 The physical features of the patient depicting eunuchoid features and delayed sexual developmental milestones

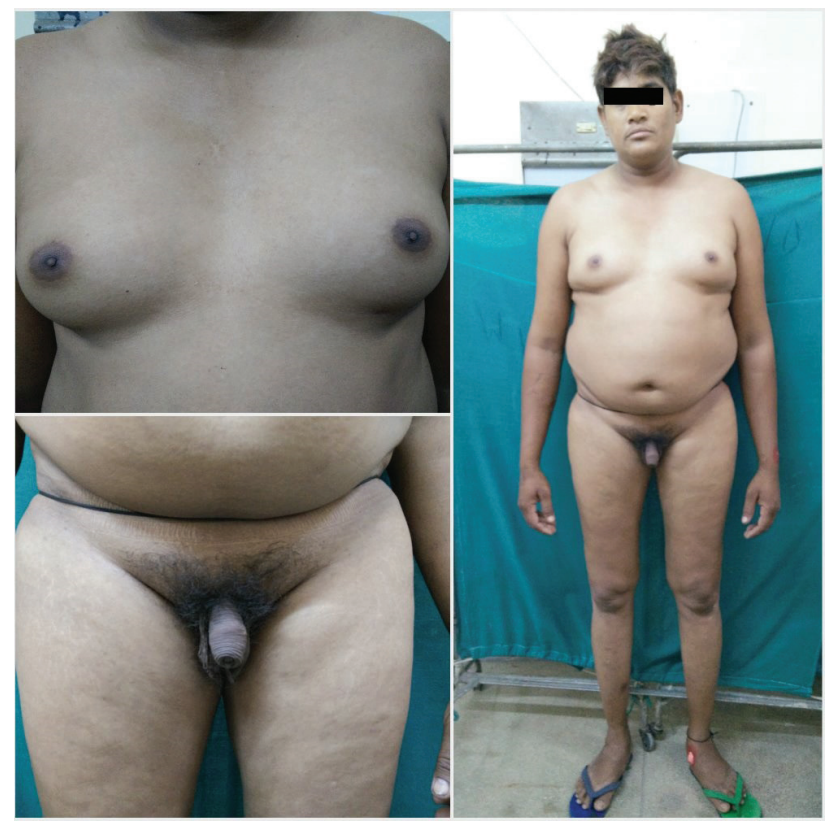

A renal biopsy which five glomeruli of which two had global glomerulosclerosis. Light microscopic examination showed endo-mesangial cellularity and normal blood vessels. Immunofluoroscence was also normal. The histopathological impression was focal global glomerulosclerosis (Figure 3).

During his hospital stay, the patient was advised on salt restriction. He was put on ramipril $5 \mathrm{mg} /$ day and eltroxin 150 $\mu \mathrm{g} /$ day. He was started on injectable testosterone therapy. He was counselled regarding his infertility and advised regarding different assisted reproduction techniques. He was also given an option of reduction mammoplasty for gynaecomastia.

During the follow up, the patient had improvement in symptoms and signs including decrease in facial puffiness, lower limb swelling and proteinuria, and increased libido. He is being continued on thyroxine and testosterone therapy with regular monitoring and monthly proteinuria quantification.

\section{Discussion}

Klinefelter's syndrome is one of the most common causes of primary hypogonadism, with an incidence of 1:500 births. It was first reported in 1942 by Klinefelter et al. and was characterised by eunuchoid appearance, gynaecomastia, small testes and increased FSH levels in urine. ${ }^{1}$ In 1959 , Jacobs and Strong reported the karyotype of the disease including several mosaic patterns. ${ }^{2}$ The disease is commonly characterised by the presence of 47 chromosomes with XXY sex karyotype.

Autoimmunity in Klinefelter's syndrome is found to be common due to the presence of an extra $X$ chromosome. The frequencies of the diseases such as Sjogren's, lupus and rheumatoid arthritis is increased due to the hormonal imbalances. ${ }^{3,4}$ In a study conducted by Seminog et al., those affected by Klinefelter's showed higher incidence of
Figure 2 Cytogenetic analysis of PHA stimulated peripheral lymphocytes revealing an extra $X$ chromosome with karyotype of $47 \mathrm{XXY}$

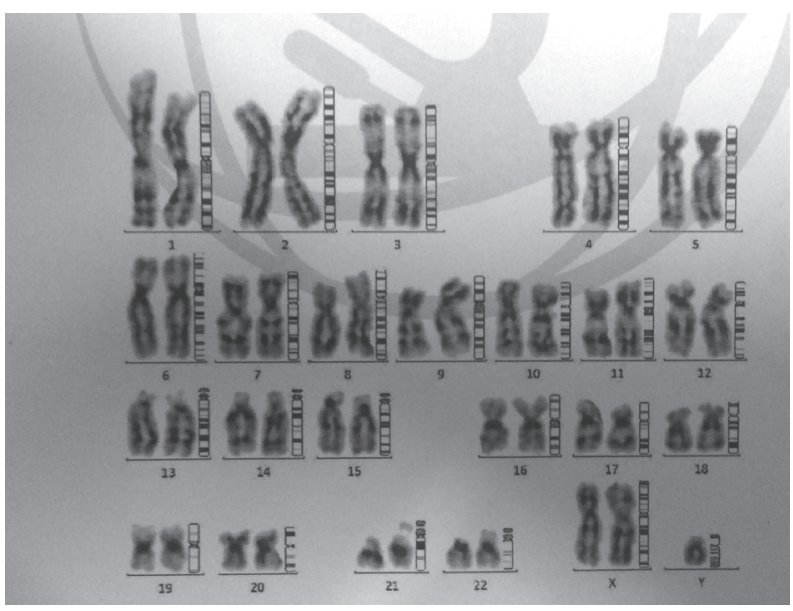

Figure 3 Renal biopsy histopathological picture showing five glomeruli of which two have global glomerulosclerosis. The histopathological impression gained was focal global glomerulosclerosis

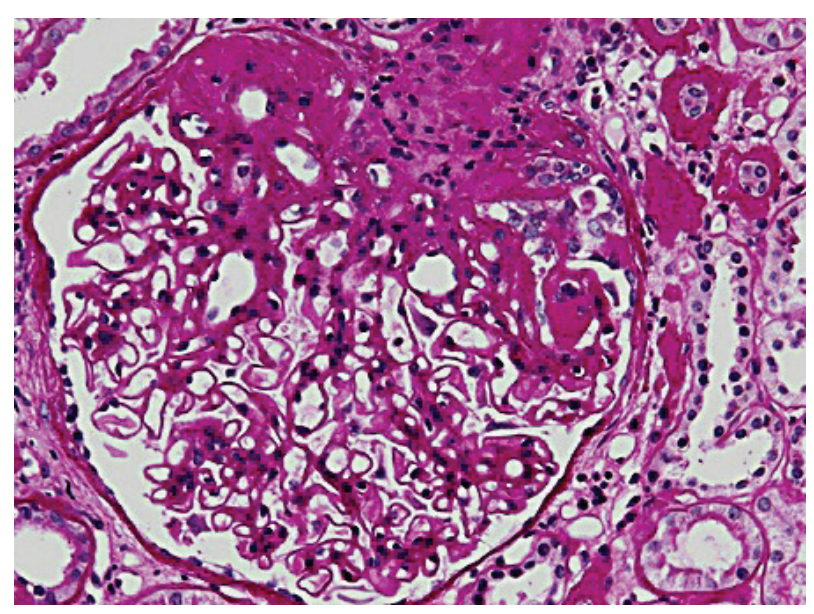

autoimmunity. ${ }^{5}$ In 2009, a study by Sawalha et al. showed that there is a gene dose effect for the $X$ chromosome. The authors hypothesised that the risk of autoimmune diseases is comparable in 45,XX women and 45,XXY men; and that the risk of autoimmunity is determined by the number of $X$ chromosome rather than the gender. ${ }^{6}$

There are very few case reports addressing the association of Klinefelter's syndrome with thyroid abnormalities. Burt et al. reported the first case of thyroid adenoma with Klinefelter's syndrome. ${ }^{7}$ In 2009, Bjorn et al. published a case control study in 75 Klinefelter's patients, concluding that there could be a general shift in fT3 towards the lower values with maintenance of normal TSH levels due to hypothalamic pituitary dysfunction as a part of Klinefelter's phenotype. ${ }^{8}$ Grand reported a case of Klinefelter's syndrome associated with pancreatic insufficiency and hypothyroidism attributed to autoimmunity. ${ }^{9}$ In the present case, the hypothyroidism was due to autoimmunity as denoted by the increased levels of anti TPO antibodies.

The novel aspect of this case is the association with nephrotic syndrome. A case of Klinefelter's syndrome 
associated with Wilms' tumour presenting as renal failure has been reported. ${ }^{10} \mathrm{An}$ association with nephrotic syndrome has not been described. Among sex chromosomal disorders, Turner's syndrome has been reported to have association with nephrotic syndrome. ${ }^{11}$ Uddin et al reported a case in which there was an association of autoimmune hypothyroidism with membranoproliferative glomerulonephritis. ${ }^{12}$ Mariani and Burts upheld the association of autoimmune hypothyroidism with nephrotic syndrome, stating membranous nephropathy, minimal change disease, membranoproliferative glomerulonephritis and IgA nephropathy were the most common associations. ${ }^{13}$ Focal glomerulonephritis has not been described with hypothyroidism. The pathogenesis of nephrotic syndrome in these cases is attributed to autoimmunity, but the exact pathway has not been defined. Saxena et al. proposed that autoimmunity might be the prime pathogenic process underlying most primary as well as secondary causes of glomerulonephritis in humans. ${ }^{14}$ In the present scenario, after ruling out alternative causes of nephrotic syndrome, we suggest that in our case it is related to the autoimmunity which is more common in Klinefelter's syndrome. (1)

\section{References}

1 Klinefelter HF Jr, Reifenstein EC Jr, Albright F. Syndrome characterized by gynecomastia, aspermatogenesis without a Leydigism and increased excretion of follicle stimulating hormone. J Clin Endocrinol 1942; 2: 615-27.

2 Jacobs PA, Strong JA. A case of human intersexuality having a possible XXY Sex-determining mechanism. Nature 1959; 83: 302-3.

3 Oslen NJ, Lovacs WJ. Case report: testosterone treatment of systemic lupus erythematosus in a patient with Klinefelter's syndrome. Am J Med Sci 1995; 310: 158-60.

4 Bizzaro A, Valentini G, Marino DI et al.Influence of testosterone therapy on clinical and immunological features of autoimmune disease associated Klinefelter's syndrome. J Clin Endocrinol Metab 1987; 64: 32-6.

5 Seminog 00 , Seminog $A B$, Yeats $D$ et al. Association between Klinefelter's syndrome and autoimmune diseases: English national record linkage studies. Autoimmunity 2015; 48: 125-8.

6 Sawalha AH, Harley JB, Scofield RH. Autoimmunity and Klinefelter's syndrome: when men have two $\mathrm{X}$ chromosomes. J Autoimmun 2009; 33: 31-4.

7 Burt AS, Reiner L, Cohen RB et al. Klinefelter's syndrome: Report of an autopsy, with particular reference to the histology and histochemistry of endocrine glands. J Clin Endocrinol Metab 1954; 14: $719-28$
8 Bjorn AM, Bojesen A, Gravholt $\mathrm{CH}$ et al. Hypothyroidism secondary to hypothalamic pituitary dysfunction may be part of the phenotype in Klinefelter's syndrome: a case control study. J Clin Endocrinol Metab 2009; 94: 2478-81.

9 Grand RJ, Rosen SW, Sant'Agnese PA et al. Unusual case of XXY Klinefelter's syndrome with pancreatic insufficiency, hypothyroidism, deafness, chronic lung disease, dwarfism and microcephaly. Am J Med 1966; 41: 478-85.

10 Benz K, Plank C, Amann K et al. Hypergonadotropic hypogonadism and renal failure due to WT1 mutation. Nephrol Dial Transplant 2006; 21: 1716-18.

11 Minzala G, Ismail G. An intriguing association of Turner syndrome with severe nephrotic syndrome: searching for a diagnosis. Lupus 2016; 25: 1266-8.

12 Uddin MD, Alam KM, Mohammed FR et al. Hypothyroidism and nephrotic syndrome - rare association. J Medicine 2009; 10: 34-5.

13 Mariani LH, Berns JS. The renal manifestations of thyroid disease. J Am Soc Nephrol 2012; 23: 22-6.

14 Saxena R, Johansson C, Bygren P et al. Autoimmunity and glomerulonephritis. Postgrad Med J 1992; 68: 242-50. 